CLINICAL STUDY

\title{
Macrophage inhibitory cytokine-1 is increased in individuals before type 2 diabetes diagnosis but is not an independent predictor of type 2 diabetes: the Whitehall II study
}

\author{
Maren Carstensen $^{1}$, Christian Herder ${ }^{1}$, Eric J Brunner ${ }^{2}$, Klaus Strassburger ${ }^{3}$, Adam G Tabak ${ }^{2,4}$, Michael Roden ${ }^{1,5}$ \\ and Daniel R Witte ${ }^{2,6}$ \\ ${ }^{1}$ Institute for Clinical Diabetology, German Diabetes Center, Leibniz Center for Diabetes Research at Heinrich Heine University Düsseldorf, Auf'm \\ Hennekamp 65, 40225 Düsseldorf, Germany, ${ }^{2}$ Department of Epidemiology and Public Health, University College London, 1-19 Torrington Place, London \\ WC1E 6BT, UK, ${ }^{3}$ Institute of Biometrics and Epidemiology, German Diabetes Center, Leibniz Center for Diabetes Research at Heinrich Heine University \\ Düsseldorf, Auf'm Hennekamp 65, 40225 Düsseldorf, Germany, ${ }^{4}$ First Department of Internal Medicine, Faculty of Medicine, Semmelweis University, \\ Korányi Sándor u. 2/a, 1083 Budapest, Hungary, ${ }^{5}$ Department of Metabolic Diseases, Heinrich Heine University Düsseldorf, Universitätsstrasse 1, \\ 40225 Düsseldorf, Germany and ${ }^{6}$ Steno Diabetes Center, Niels Steensens Vej 2-4, 2820 Gentofte, Denmark
}

(Correspondence should be addressed to C Herder; Email: christian.herder@ddz.uni-duesseldorf.de)

\begin{abstract}
Objective: Macrophage inhibitory cytokine-1 (MIC-1) belongs to the transforming growth factor (TGF)- $\beta$ superfamily, and has been reported to be involved in energy homoeostasis and weight loss and to have anti-inflammatory properties. We hypothesized that decreased concentrations of MIC-1 would be associated with higher risk of developing type 2 diabetes.

Design and methods: We designed a nested case-control study within the Whitehall II cohort and measured serum concentrations of MIC-1 by ELISA in 180 individuals without type 2 diabetes at baseline who developed type 2 diabetes during the follow-up period of $11.5 \pm 3.0$ years and in 372 controls frequency-matched for age, sex, and body mass index with normal glucose tolerance throughout the study.

Results: MIC-1 concentrations at baseline were higher in cases (median (25/75th percentiles) 537.1 $(452.7-677.4) \mathrm{pg} / \mathrm{ml})$ than in controls $(499.7(413.8-615.4) \mathrm{pg} / \mathrm{ml} ; P=0.0044)$. In the age- and sex-adjusted model, a 1-S.D. increase in MIC-1 $(206.0 \mathrm{pg} / \mathrm{ml})$ was associated with an odds ratio ( $95 \%$ confidence interval) of $1.21(0.997 ; 1.46 ; P=0.054)$ for type 2 diabetes. Adjustment for waist circumference, cardiovascular risk factors, socioeconomic status, proinflammatory mediators, and glycemia abolished the association.

Conclusions: Baseline MIC-1 concentrations were increased, not decreased, in individuals before type 2 diabetes manifestation, but not independently associated with incident type 2 diabetes in multivariable analyses. This upregulation of MIC-1 could be part of an anti-inflammatory response preceding the onset of type 2 diabetes, which has been described before for interleukin-1 receptor antagonist and TGF- $\beta 1$.
\end{abstract}

European Journal of Endocrinology 162 913-917

\section{Introduction}

Serum levels of several pro- and anti-inflammatory cytokines are elevated in individuals who will subsequently develop type 2 diabetes $(1,2)$, and so far adiponectin is the only adipokine for which an inverse association of serum levels with risk for type 2 diabetes has been described (3). Macrophage inhibitory cytokine-1 (MIC-1), a member of the transforming growth factor (TGF)- $\beta$ superfamily (4), has emerged as a candidate anti-inflammatory link due to its regulatory role in energy homoeostasis with elevated levels being associated with weight loss (5). MIC-1 is expressed in several tissues throughout the body including liver, kidney, and adipose tissue (6). Although MIC-1 is expressed in adipose tissue and secreted from adipocytes, an inverse correlation between MIC-1 gene expression and measures of obesity has been reported (6). Basic physiological research also points to an inverse association between proinflammatory cytokines and MIC-1. MIC-1 gene expression is downregulated by leptin and interleukin (IL)-1 $\beta$, whereas MIC-1 increases the release of adiponectin from adipocytes (6), and attenuates lipopolysaccharide-induced tumor necrosis factor- $\alpha$ release from macrophages (4). Macrophages have been implicated in different aspects of diabetes development. Several studies reported an increased number of macrophages in pancreatic islets from patients with type 2 diabetes (7) and in adipose tissue of obese individuals $(8-10)$ as well as higher activation 
of peripheral blood monocytes in patients with poorly controlled diabetes (11). Activated macrophages secrete proinflammatory and chemotactic cytokines and chemokines that can impair $\beta$-cell function and adipocyte insulin sensitivity, and stimulate further activation and infiltration of monocytes into these tissues (1).

Data from prospective studies on MIC-1 and risk for type 2 diabetes are not yet available. A small crosssectional study recently reported elevated, not decreased, serum levels of MIC-1 in obesity and type 2 diabetes (12). However, based on the aforementioned reports that pointed toward anti-inflammatory properties of MIC-1, we hypothesized that MIC-1 could be regulated in a similar manner as adiponectin, and that increased serum concentrations would be associated with decreased risk for type 2 diabetes. The aims of this study were to examine the relationship of MIC-1 serum concentration with anthropometric, metabolic, and inflammatory variables and to characterize its association with incident type 2 diabetes.

\section{Methods}

\section{Study design}

The presented data are from a nested case-control study within the Whitehall II cohort. The Whitehall II study started in 1985 and included 10308 civil servants aged 35-55 years in phase 1 (1985-1988). A detailed description of the cohort profile was given previously (13).

The baseline for the current study was phase 3 $(n=7537)$ (1991-1994) as this was the first phase including a $75 \mathrm{~g}$ oral glucose tolerance test (OGTT). In phases 4-8, participants were followed through postal questionnaires at $\sim 2$.5-year intervals. Clinical examinations including an OGTT were additionally performed at phases 3,5 , and 7 .

All participants selected for the current nested case-control study $(n=552)$ were diabetes free at baseline (phase 3 ). Individuals who developed type 2 diabetes during the follow-up period of $11.5 \pm 3.0$ years served as cases $(n=180)$. Controls $(n=372)$ with normal glucose tolerance at baseline and during follow-up were frequency-matched to cases for age (5-year bands), sex, and body mass index (BMI; $5 \mathrm{~kg} / \mathrm{m}^{2}$ bands). The study population is slightly smaller than the sample from a previous report (2) as no serum for MIC-1 assays was available for seven study participants.

Individuals with prevalent or incident cardiovascular disease, incident coronary heart disease, self-reported longstanding inflammatory illness, recent inflammatory symptoms, anti-inflammatory medication, and/or non-white ethnicity were excluded.

\section{Experimental and statistical analyses}

Serum concentrations of MIC-1 (growth differentiation factor-15, GDF-15) were measured with the Quantikine Human GDF-15 Immunoassay kit (R\&D Systems, Wiesbaden, Germany). Mean intra-assay and interassay coefficients of variation were 2.7 and $3.3 \%$ respectively. The limit of detection was $23.4 \mathrm{pg} / \mathrm{ml}$. All samples gave values above the limit of detection.

Univariate associations of MIC-1 with anthropometric, metabolic, and immunological variables were estimated using linear regression. Logistic regression was used to assess the association between MIC-1 levels, possible covariates and incident type 2 diabetes. Results are given as odds ratios (OR) and corresponding 95\% confidence intervals $(95 \% \mathrm{CI})$. For all statistical analyses, $P<0.05$ was considered to be statistically significant. All statistical analyses were performed with the statistical computer software SAS (version 9.2, SAS Institute, Cary, NC, USA).

\section{Results}

\section{Study population}

Characteristics of an almost identical study population have been described before (2), and baseline characteristics of participants stratified in controls $(n=372)$ and cases $(n=180)$ are shown in Supplementary Table 1 (see section on supplementary data given at the end of this article). Cases had higher waist circumference (men only), blood pressure, serum levels of fasting triglycerides, fasting and 2-h glucose as well as fasting insulin, higher serum concentrations of MIC-1, IL-1Ra and C-reactive protein (CRP) compared with the controls. Moreover, cases had lower insulin sensitivity, and were more frequently on antihypertensive medication.

\section{Cross-sectional associations of MIC-1 with anthropometric, metabolic, and immunological variables}

Age showed a strong positive association with serum MIC-1 concentrations, and weaker associations were present with BMI, waist circumference, blood pressure, total cholesterol, and fasting triglycerides (Table 1). Fasting insulin and homeostasis model assessment of insulin resistance were positively associated with MIC-1, whereas neither fasting nor 2-h blood glucose was associated with MIC-1. Regarding pro- and antiinflammatory immune mediators, CRP, IL-6, and IL-1Ra, but not adiponectin, showed a positive association with MIC-1. In subanalyses, associations tended to be stronger among cases than controls. 
Table 1 Association between baseline serum macrophage inhibitory cytokine-1 $\left(\log _{2}\right.$ transformed) and anthropometric, metabolic, and immunological variables.

\begin{tabular}{|c|c|c|c|}
\hline Variables & $\begin{array}{c}\text { Controls }(n=372) \\
\beta(95 \% \mathrm{Cl})\end{array}$ & $\begin{array}{c}\text { Cases }(n=180) \\
\beta(95 \% \mathrm{Cl})\end{array}$ & $\begin{array}{c}\text { All }(n=552) \\
\beta(95 \% \mathrm{Cl})\end{array}$ \\
\hline \multicolumn{4}{|l|}{ Anthropometric factors } \\
\hline Age (years) & $0.025(0.018 ; 0.032)^{\ddagger}$ & $0.034(0.022 ; 0.045)^{\ddagger}$ & $0.028(0.022 ; 0.034)^{\ddagger}$ \\
\hline $\mathrm{BMI}\left(\mathrm{kg} / \mathrm{m}^{2}\right)$ & $0.008(-0.004 ; 0.020)$ & $0.024(0.008 ; 0.039)^{\dagger}$ & $0.016(0.007 ; 0.026)^{\ddagger}$ \\
\hline Waist circumference $(\mathrm{cm})$ & $0.003(-0.001 ; 0.007)$ & $0.011(0.005 ; 0.016)^{\ddagger}$ & $0.006(0.003 ; 0.010)^{\ddagger}$ \\
\hline Systolic blood pressure $(\mathrm{mmHg})$ & $0.003(-0.001 ; 0.006)$ & $0.008(0.004 ; 0.013)^{\ddagger}$ & $0.005(0.003 ; 0.008)^{\ddagger}$ \\
\hline Diastolic blood pressure (mmHg) & $0.004(-0.001 ; 0.009)$ & $0.009(0.002 ; 0.015)^{\star}$ & $0.006(0.002 ; 0.010)^{\dagger}$ \\
\hline \multicolumn{4}{|l|}{ Metabolic factors } \\
\hline Total cholesterol $(\mathrm{mmol} / \mathrm{l})$ & $0.043(0.004 ; 0.081)^{*}$ & $0.063(0.001 ; 0.124)^{*}$ & $0.054(0.021 ; 0.086)^{\dagger}$ \\
\hline Fasting triglycerides $(\mathrm{mmol} / \mathrm{l})$ & $0.090(0.035 ; 0.145)^{\dagger}$ & $0.128(0.042: 0.215)^{\dagger}$ & $0.113(0.067 ; 0.159)^{\ddagger}$ \\
\hline Fasting glucose $(\mathrm{mmol} / \mathrm{l})$ & $0.068(-0.060 ; 0.196)$ & $0.015(-0.125 ; 0.154)$ & $0.061(-0.025 ; 0.147)$ \\
\hline 2-h glucose $(\mathrm{mmol} / \mathrm{l})$ & $0.005(-0.037 ; 0.046)$ & $0.011(-0.029 ; 0.052)$ & $0.023(-0.002 ; 0.048)$ \\
\hline Fasting insulin (mU/l) & $0.047(-0.005 ; 0.099)$ & $0.126(0.046 ; 0.205)^{\dagger}$ & $0.084(0.042 ; 0.126)^{\ddagger}$ \\
\hline HOMA-IR & $0.048(-0.003 ; 0.099)$ & $0.119(0.042 ; 0.195)^{\dagger}$ & $0.081(0.041 ; 0.121)^{\ddagger}$ \\
\hline \multicolumn{4}{|l|}{ Immunological factors } \\
\hline CRP (mg/l) & $0.071(0.041 ; 0.102)^{\ddagger}$ & $0.068(0.026 ; 0.110)^{\dagger}$ & $0.075(0.051 ; 0.100)^{\ddagger}$ \\
\hline IL-6 (pg/ml) & $0.121(0.060 ; 0.181)^{\ddagger}$ & $0.149(0.049 ; 0.249)^{\dagger}$ & $0.135(0.082 ; 0.187)^{\ddagger}$ \\
\hline IL-1Ra $(\mathrm{pg} / \mathrm{ml})$ & $0.096(0.020 ; 0.172)^{\star}$ & $0.157(0.059 ; 0.256)^{\dagger}$ & $0.141(0.083 ; 0.200)^{\ddagger}$ \\
\hline Adiponectin $(\mathrm{ng} / \mathrm{ml})^{\mathrm{a}}$ & $-0.0026(-0.107 ; 0.102)$ & $-0.096(-0.216 ; 0.024)$ & $-0.051(-0.129 ; 0.026)$ \\
\hline
\end{tabular}

Data are from linear regression analysis, and are given as $\beta$ values, 95\% $\mathrm{Cl}$, and corresponding $P$ values. Triglycerides, insulin, HOMA-IR, C-reactive protein (CRP), interleukin (IL)-6, IL-1Ra and adiponectin entered the models as $\log _{2}$-transformed variables. ${ }^{\star} P<0.05 ;{ }^{\dagger} P<0.01 ;{ }^{\ddagger} P<0.001$.

${ }^{a}$ Adiponectin concentrations were available for a subset of study participants (158 controls and 139 cases).

\section{MIC-1 and incident diabetes}

Table 2 shows the association between circulating concentrations of MIC-1 and incident diabetes. MIC-1 concentrations at baseline were higher in cases (median $537.1 \mathrm{pg} / \mathrm{ml}(25-75$ th percentiles $452.7-677.4))$ than in controls (499.7 (413.8-615.4); $P=0.0044)$. In the age- and sex-adjusted model, the association between a 1-S.D. increase of MIC-1 $(206.0 \mathrm{pg} / \mathrm{ml})$ and increased risk for type 2 diabetes was borderline significant (OR (95\% CI) 1.21 (0.997; 1.46)). Additional adjustment for waist circumference, cardiovascular risk factors, socioeconomic status, proinflammatory mediators, or glycemia abolished the association.

\section{Discussion}

Our nested case-control study within the Whitehall II study is the first prospective investigation of the association between MIC-1 serum concentrations and incident type 2 diabetes. We found that MIC-1 levels were significantly higher in individuals who subsequently developed type 2 diabetes than those who remained diabetes free. However, there was only a borderline significant association between MIC-1 and incident type 2 diabetes after adjustment for age and sex, which was further attenuated after adjustment for waist circumference, cardiovascular risk factors, socioeconomic status, proinflammatory mediators,

Table 2 Association between circulating concentrations of macrophage inhibitory cytokine-1 at baseline and incident type 2 diabetes.

\begin{tabular}{|c|c|c|c|}
\hline Model & Covariables & OR $(95 \% \mathrm{Cl})$ & $\boldsymbol{P}$ \\
\hline 1 & Age, sex & $1.21(0.997 ; 1.46)$ & 0.054 \\
\hline 2 & Age, sex, and waist circumference & $1.15(0.95 ; 1.40)$ & 0.16 \\
\hline 3 & $\begin{array}{l}\text { Model } 2+\text { cardiovascular risk factors (cholesterol, fasting triglycerides, blood } \\
\text { pressure, smoking }{ }^{\mathrm{a}} \text {, physical activity }{ }^{\mathrm{b}} \text {, antihypertensive medication, } \\
\text { and lipid-lowering medication) }\end{array}$ & $1.06(0.86 ; 1.30)$ & 0.58 \\
\hline 4 & Model $2+$ socioeconomic status (employment grade $^{c}$ ) & $1.11(0.91 ; 1.35)$ & 0.29 \\
\hline 5 & Model 2+ proinflammatory mediators (CRP; IL-6) & $1.12(0.92 ; 1.38)$ & 0.26 \\
\hline 6 & Model $2+$ glycemia (fasting glucose, 2 -h glucose and fasting insulin) & $1.12(0.88 ; 1.42)$ & 0.35 \\
\hline 7 & Combination of all covariables from models $2-6$ & $1.05(0.78 ; 1.41)$ & 0.75 \\
\hline
\end{tabular}

Data are given as odds ratio (95\% Cl) for a 1-s.D. increase $(206.0 \mathrm{pg} / \mathrm{ml})$ of MIC-1 concentrations. Triglycerides, C-reactive protein (CRP), interleukin (IL)-6, and insulin entered the models as In-transformed variables.

${ }^{a}$ Smoking is coded in three classes (never smoked, former smoker, and current smoker).

bPhysical activity is coded in three classes (none/mild, moderate, and vigorous).

${ }^{c}$ Employment grade is coded in six classes running from 1 (highest grade) to 6 (lowest grade). 
and glycemia. This reduction in the effect size is in line with the positive associations we observed between MIC-1 and potential confounders which are known risk factors for type 2 diabetes.

Our main finding does not support the hypothesis that MIC-1 could be, through its anti-inflammatory effects, a protective cytokine against the development of type 2 diabetes. Our data extend current knowledge on the role of MIC-1 in the development of type 2 diabetes. So far, there is only one report from a small crosssectional study including 54 women that reported increased concentrations of MIC-1 in patients with type 2 diabetes (12). Due to its cross-sectional design, it could not exclude the possibility that elevated concentrations of $\mathrm{MIC}-1$ were a consequence and not a cause of type 2 diabetes. We demonstrate that MIC-1 is already increased over 11 years before the manifestation of type 2 diabetes.

MIC-1 may be a part of a counterregulatory, antiinflammatory response which is independently regulated from adiponectin. We used the same study sample before to show that serum levels of the antiinflammatory cytokine IL-1Ra were elevated before the diagnosis of type 2 diabetes (2). The concept of the presence of an anti-inflammatory counterregulation before type 2 diabetes is further supported by data from the MONICA/KORA study that demonstrates an association between elevated serum levels of the anti-inflammatory and immunosuppressive cytokine TGF- $\beta 1$ and increased risk of type 2 diabetes (14). Taken together, these findings regarding type 2 diabetes should be seen in context with the general regulatory principle in inflammation that in acute conditions, proinflammatory stimuli at the beginning of the process induce anti-inflammatory mediators in order to resolve inflammation (15). In individuals with an increased risk for type 2 diabetes, a chronic state of immune activation can be found. The concomitant and persistent upregulation of both pro-and anti-inflammatory cytokines in these individuals may reflect their inability to efficiently counterregulate inflammatory stimuli (16).

Our data indicate that despite higher levels in cases than in controls in the unadjusted analysis, MIC- 1 by itself is not associated with the risk for type 2 diabetes independently of established risk factors. It is interesting to note that circulating levels of MIC-1 have been found to be independent predictors of cardiovascular events and mortality (17-19). There are some putative explanations why our findings were contrary to our hypothesis. In the study by Johnen et al. (5), the association between higher MIC-1 and lower BMI was consistent across several mouse models, whereas associations with low BMI and weight loss in man were detected in patient samples with prostate cancer or chronic renal failure which clearly differ from our study population of initially healthy middle-aged men and women. Moreover, we did not confirm the previously observed inverse correlation between MIC-1 gene expression and BMI (6). However, this finding was based on measurements with MIC-1 mRNA in adipose tissue from small study samples with a very wide BMI range $\left(\sim 20-80 \mathrm{~kg} / \mathrm{m}^{2}\right)$, whereas our data represent a much lower average BMI. In addition, we measured MIC-1 protein concentrations in the circulation which we believe to be an integrated measure of MIC-1 secretion and turnover in many body tissues.

Our study has several limitations and strengths. Point estimates and CIs are derived from non-weighted data from a nested case-control study and therefore, statistical inferences based upon them may be restricted and may not represent the best available estimate within the context of the original cohort. Furthermore, the cohort consists of employees of the British Civil Service who were mainly male and middle-aged. However, this is, to the best of our knowledge, the first prospective study to analyze whether MIC-1 serum levels were associated with incident type 2 diabetes. In addition, our analyses are based on a wellphenotyped cohort.

In conclusion, we found that increased MIC-1 levels were not independently associated with incident type 2 diabetes, although MIC-1 levels were elevated in individuals before type 2 diabetes manifestation in the unadjusted analysis.

\section{Supplementary data}

This is linked to the online version of the paper at http://dx.doi.org/ 10.1530/EJE-09-1066.

\section{Declaration of interest}

The authors declare that there is no conflict of interest that could be perceived as prejudicing the impartiality of the research reported.

\section{Funding}

The Whitehall II study is funded by the Medical Research Council; the Economic and Social Research Council; the British Heart Foundation; the Health and Safety Executive; the Department of Health (all UK); the National Heart Lung and Blood Institute (HL36310), NIH; the National Institute on Aging (AG13196), NIH; the Agency for Health Care Policy Research (HSO6516); and the John and Catherine MacArthur Foundation. This case-control study was funded by a Medical Research Council New Investigator Grant (G0501184), the Federal Ministry of Health (Berlin, Germany), and the Ministry of Innovation, Science, Research and Technology of the state North Rhine-Westphalia (Düsseldorf, Germany).

\section{References}

1 Kolb H \& Mandrup-Poulsen T. An immune origin of type 2 diabetes? Diabetologia 200548 1038-1050.

2 Herder C, Brunner EJ, Rathmann W, Strassburger K, Tabak AG, Schloot NC \& Witte DR. Elevated levels of the anti-inflammatory interleukin-1 receptor antagonist precede the onset of type 2 diabetes. Diabetes Care 200932 421-423.

3 Li S, Shin HJ, Ding EL \& von Dam RM. Adiponectin levels and risk of type 2 diabetes: a systematic review and meta-analysis. Journal of the American Medical Association 2009302 179-188. 
4 Bootcov MR, Bauskin AR, Valenzuela SM, Moore AG, Bansal M, He XY, Zhang HP, Donnellan M, Mahler S, Pryor K, Walsh BJ, Nicholson RC, Fairle WD, Por SB, Robbins JM \& Breit SN. MIC-1, a novel macrophage inhibitory cytokine, is a divergent member of the TGF-beta superfamily. PNAS 199794 11514-11519.

5 Johnen H, Lin S, Kuffner T, Brown DA, Tsai VW, Bauskin AR, Wu L, Pankhurst G, Jiang L, Junankar S, Hunter M, Fairlie WD, Lee NJ, Enriquez RF, Baldock PA, Corey E, Apple FS, Murakami MM, Lin EJ, Wang C, During MJ, Sainsbury A, Herzog H \& Breit SN. Tumor-induced anorexia and weight loss are mediated by the TGF-beta superfamily cytokine MIC-1. Nature Medicine 200713 1333-1340.

6 Ding Q, Mracek T, Gonzalez-Muniesa P, Kos K, Wilding J, Trayhurn $\mathrm{P} \&$ Bing $\mathrm{C}$. Identification of macrophage inhibitory cytokine- 1 in adipose tissue and its secretion as an adipokine by human adipocytes. Endocrinology $20091501688-1696$.

7 Ehses JA, Perren A, Eppler E, Ribaux P, Pospisilik JA, Maor-Cahn R, Gueripel X, Ellingsgaard H, Schneider MKJ, Biollaz G, Fontana A, Reinecke M, Homo-Delarche F \& Donath MY. Increased number of islet-associated macrophages in type 2 diabetes. Diabetes $2007 \mathbf{5 6}$ 2356-2370.

8 Weisberg SP, McCann D, Desai M, Rosenbaum M, Leibel RL \& Ferrrante AW Jr. Obesity is associated with macrophage accumulation in adipose tissue. Journal of Clinical Investigation $20031121796-1808$.

$9 \mathrm{Xu} \mathrm{H}$, Barnes GT, Yang Q, Tan G, Yang D, Chou CJ, Sole J, Nichols A, Ross JS, Tartaglia LA \& Chen H. Chronic inflammation in fat plays a crucial role in the development of obesityrelated insulin resistance. Journal of Clinical Investigation 2003 112 1821-1830.

10 Curat CA, Miranville A, Sengenes C, Diehl M, Tonus C, Busse R \& Bouloumie A. From blood monocytes to adipose tissue - resident macrophages induction of diapedesis by human mature adipocytes. Diabetes 200453 1285-1292.

11 Cipolletta C, Ryan KE, Hanna EV \& Trimble ER. Activation of peripheral blood $\mathrm{CD}^{+} 4^{+}$monocytes occurs in diabetes. Diabetes $2005542779-2786$.
12 Dostalova I, Roubicek T, Bartlova M, Mraz M, Lacinova Z, Haluzikova D, Kavalkova P, Matoulek M, Kasalicky M \& Haluzik M. Increased serum concentrations of macrophage inhibitory cytokine- 1 in patients with obesity and type 2 diabetes mellitus: the influence of very low caloric diet. European Journal of Epidemiology $2009161397-404$.

13 Marmot M \& Brunner E. Cohort profile: the Whitehall II study. International Journal of Epidemiology 200534 251-256.

14 Herder C, Zierer A, Koenig W, Roden M, Meising C \& Thorand B. Transforming growth factor-beta1 and incident type 2 diabetes: results from the MONICA/KORA case-cohort study, 1984-2002. Diabetes Care 200932 1921-1923.

15 Serhan CN \& Savill J. Resolution of inflammation: the beginning programs the end. Nature Immunology 20056 1191-1197.

16 Kolb H \& Mandrup-Poulsen T. The global diabetes epidemic as a consequence of lifestyle-induced low-grade inflammation. Diabetologia 201053 10-20.

17 Brown DA, Breit SN, Buring J, Fairlie WD, Bauskin AR, Lin T \& Ridker PM. Concentration in plasma of macrophage inhibitory cytokine-1 and risk of cardiovascular events in women: a nested case-control study. Lancet 2002359 2159-2163.

18 Wollert KC, Kempf T, Lagerqvist B, Lindahl B, Olofsson S, Allhoff T, Peter T, Siegbahn A, Venge P, Drexler H \& Wallentin L. Growth differentiation factor 15 for risk stratification and selection of an invasive treatment strategy in non ST-elevation acute coronary syndrome. Circulation 2007116 1540-1548.

19 Kempf T, Sinning J-M, Quint A, Bickel C, Sinning C, Wild PS, Schnabel R, Lubos E, Rupprecht HJ, Münzel T, Drexler H, Blankenberg S \& Wollert K. Growth-differentiation factor-15 for risk stratification in patients with stable and unstable coronary heart disease: results from the AtheroGene study. Circulation. Cardiovascular Genetics 20092 286-292.

Received 2 February 2010

Accepted 17 February 2010 